\title{
Diffusion-Weighted and Intravoxel Incoherent Motion MRI of the pregnant cervix as a tool to monitor cervical ripening
}

\author{
Adam Warner, BS ${ }^{1}$, Gary Hutchins, $\mathrm{PhD}^{2}$, Yu-Chien Wu, MD PhD², Brandon \\ Brown, MD², Monica Forbes-Amrhein, MD PhD² \\ ${ }^{1}$ Indiana University School of Medicine, ${ }^{2}$ Indiana University School of Medicine, \\ Department of Radiology and Imaging Sciences
}

\begin{abstract}
Background and Hypothesis: Preterm delivery is a major source of infant morbidity and mortality and is difficult to predict. The process of cervical ripening prior to delivery has known histologic changes including breakdown of collagen and increasing water content. It is hypothesized that the diffusion of water, which can be measured on MRI, will increase as the cervical water content increases. Diffusion weighted imaging (DWI) is a form of MRI that measures the random Brownian motion of water molecules (represented as the apparent diffusion coefficient (ADC)). Intravoxel incoherent motion (IVIM) MRI further subdivides the ADC into microcapillary perfusion $\left(D^{*}\right)$ and diffusion (D). We hypothesize that as the pregnant cervix ripens near delivery, diffusion of water within the cervix (ADC and $D$ ) will increase without changes in the capillary perfusion $\left(D^{*}\right)$. We sought to determine the relationship between $A D C, D^{*}, D$, gestational age, and time to delivery in a cohort of volunteer pregnant females.
\end{abstract}

Experimental Design or Project Methods: DWI and IVIM MR studies from a cohort of 45 volunteer pregnant females with no known underlying fetal anomalies were examined. Subglandular and stromal cervix thickness and cervical length were measured along with the ADC, $D^{*}$, and $D$ of the subglandular and stromal cervix. Gestational history and delivery information was documented.

Results: Subglandular ADC inversely correlated with time to delivery $(r=-0.393$, $p=0.052$ ). Although not significant, subglandular $D$ revealed a trend of increasing with increased gestation age $(r=0.261, p=0.149)$. Subglandular ADC also varied inversely with thickness of the subglandular cervix $(r=-0.352, p=0.047)$. Subglandular $D^{*}$ varied inversely with maternal age $(r=-0.380, p=0.028)$.

Conclusion and Potential Impact: Our data support the hypothesized trend of increased diffusion of water within the cervix with unchanged capillary perfusion as a normal pregnancy progressed throughout the second and third trimesters. This study suggests that diffusion measures (ADC and D) follow a predictable progression during the course of a normal pregnancy and have the potential to provide a means of predicting preterm labor in the setting of preterm cervical ripening. 